Pacific Journal of Mathematics

A CHARACTERIZATION OF COVERING DIMENSION BY USE 


\title{
A CHARACTERIZATION OF COVERING DIMENSION BY USE OF $\Delta_{k}(X)$
}

\author{
Jeroen BruiJning and Jun-ITi Nagata
}

\begin{abstract}
Covering dimension, in the sense of Katětov, of a topological space $X$ is characterized by use of $\Delta_{k}(X)$ which will be defined in the main discussion in terms of cardinalities of finite open covers of $X$.
\end{abstract}

1. Introduction. L. Pontrjagin and L. Schnirelmann [6] characterized dimension of a compact metrizable space $X$ by use of the numbers $N_{\rho}(\varepsilon, X)=\min \{m \in N \mid$ the metric space $(X, \rho)$ has a cover $\mathscr{U}$ such that $|\mathscr{C}|=m$ and $\operatorname{diam} U \leqq \varepsilon$ for every $U \in \mathscr{U}\}$. Their result is quite interesting in the sense that covering dimension, which is defined in terms of order (a kind of local cardinality) of a cover, is characterized in terms of global cardinality of a cover. J. Bruijning [1] generalized Pontrjagin-Schnirelmanns theorem to separable metric spaces by use of totally bounded metrics and to topological spaces by means of totally bounded pseudometrics.

In the present paper, we shall characterize covering dimension of topological spaces by use of a new function $\Delta_{k}(X)$, which will be defined later. It seems that $\Delta_{k}(X)$ can provide us with a neater characterization of dimension, perhaps because it does not involve the metric $\rho$ in its definition while $N_{\rho}(\varepsilon, X)$ does.

2. Conventions, In the following discussions we frequently consider a finite collection $\mathscr{C}=\left\{U_{1}, \cdots, U_{i}\right\}$ of subsets of a space $X$ such that $\bigcup\left\{U_{j} \mid 1 \leqq j \leqq i\right\} \supset A$ for a certain subset $A$ of $X$, and a cover $\mathscr{V}=\left\{V_{1}, \cdots, V_{i}\right\}$ of $A$ such that $V_{j} \subset U_{j} \cap A$ for $1 \leqq j \leqq i$. Then we may say: $\mathscr{U}$ is shrunk to $\mathscr{V}$ on $A$.

If $\mathscr{V}$ consists of open (closed) subsets of $A$, we shrink $\mathscr{U}$ to the open (closed) cover of of $A$. If $A=X$, we may drop the words "on $A$ ". If $\cap \mathscr{V}=\varnothing, \mathscr{V}$ is vanishing.

We shall denote by $C_{m}^{k}$ the set of all $m$-element subsets of the set $\{1,2, \cdots, k\}$ and by $\left(\begin{array}{c}k \\ m\end{array}\right)$ its cardinality, i.e., $\left(\begin{array}{l}k \\ m\end{array}\right)=k ! / m !(k-m)$ !.

By the dimension of a space $X, \operatorname{dim} X$, we will mean its Katětov dimension, i.e.,

$$
\begin{array}{ll}
\operatorname{dim} X=-1 & \text { iff } \quad X=\varnothing ; \\
\operatorname{dim} X \leqq n(n \geqq 0) & \text { iff every finite cover of } X,
\end{array}
$$

consisting of functionally open sets, has a finite refinement, also consisting of functionally open sets and with order $\leqq n+1$; 
$\operatorname{dim} X=n(n \geqq 0) \quad$ iff $\quad \operatorname{dim} X \leqq n$ but not $\operatorname{dim} X \leqq n-1$;

$\operatorname{dim} X=\infty \quad$ iff $\operatorname{not} \operatorname{dim} X \leqq n$, for every $n$.

We will sometimes use the following, without explicitly mentioning it: for normal spaces, Katētov dimension coincides with ordinary covering dimension [3, p. 268].

For basic concepts in general topology and dimension theory see [3], [4], and [5]. The reader is warned that different authors sometimes mean different numbers by the order of a cover; in our definition, the order is the maximum number of mutually intersecting sets in the cover, but in Engelking [3] the order is defined to be one less. Since we will frequently be referring to [3] the reader should be aware of this.

3. The main theorem: the normal case. Let $X$ be a topological space and $k$ a positive integer. Define $\Delta_{k}(X)=\min \{m \in N \mid$ for every functionally open cover $\mathscr{C}$ of $X$ with $|\mathscr{U}| \leqq k$ there exists a functionally open cover $\mathscr{V}$ of $X$ with $|\mathscr{V}| \leqq m$ and $\left.\mathscr{V}^{\lrcorner}<\mathscr{U}\right\}$.

Here $\mathscr{\mathscr { C }}^{j}=\{\operatorname{St}(x, \mathscr{\mathscr { C }}) \mid x \in X\}$ and $<$ means: "refines".

REMARK. If $X$ is a normal space, we may drop the word "functionally" in the above definition either or both times it occurs and still arrive at the same number. This is easily proved using similar techniques as in the proof that Katẽtov dimension coincides with ordinary covering dimension referred to above. We will use this observation in the sequel without explicitly mentioning it.

Proposition 1. Let $n \geqq 0$, and $X$ be a topological space with $\operatorname{dim} X \leqq n$. Let $k \in N$. Then

$$
\begin{array}{ll}
\Delta_{k}(X) \leqq 2^{k}-1 & \text { if } k \leqq n+1 \\
\Delta_{k}(X) \leqq\left(\begin{array}{l}
k \\
1
\end{array}\right)+\cdots+\left(\begin{array}{c}
k \\
n+1
\end{array}\right) \text { if } k \geqq n+1 .
\end{array}
$$

Proof. Suppose $\mathscr{C}=\left\{U_{1}, \cdots, U_{k}\right\}$ is a functionally open cover of $X$. Since $\operatorname{dim} X \leqq n$, we can shrink $\mathscr{C}$ to a functionally open cover $\mathscr{V}=\left\{V_{1}, \cdots, V_{l k}\right\}$ with ord $\mathscr{V} \leqq n+1$. Further shrink $\mathscr{V}$ to a functionally closed cover $\mathscr{F}=\left\{F_{1}, \cdots, F_{k}\right\}$ (as in [3, p. 267]). For every nonempty $A \subset\{1, \cdots, k\}$ we define the following functionally open set:

$$
W(A)=\left[\cap\left\{V_{i} \mid i \in A\right\}\right] \cap\left[\cap\left\{X \backslash F_{i} \mid i \notin A\right\}\right] .
$$

Let $\mathscr{W}=\{W(A) \mid W(A) \neq \varnothing\}$. Since ord $\mathscr{V} \leqq n+1, W(A)=\varnothing$ if $|A|>$ 
$n+1$. Therefore $|\mathscr{W}| \leqq 2^{k}-1$ if $k \leqq n+1$, and $|\mathscr{W}| \leqq\left(\begin{array}{c}k \\ 1\end{array}\right)+\cdots$ $+\left(\begin{array}{c}k \\ n+1\end{array}\right)$ if $k \geqq n+1$. It is also easy to see that $\mathscr{W}^{\Delta}<\mathscr{P}<\mathscr{C}$, because for each $x \in F_{i}$, St $\left(x, \mathscr{W}^{-}\right) \subset V_{i}$. This proves Proposition 1.

Lemma 1. Let $X$ be a normal space and $n \geqq 0$. Then $\operatorname{dim} X \leqq n$ iff every open cover $\left\{W_{1}, \cdots, W_{n+2}\right\}$ of $X$ can be shrunk to a vanishing open cover of $X$.

Proof. See Engelking [3, p. 282].

LEMMA 2. Let $X$ be a normal space with $\operatorname{dim} X \geqq n$, where either $n \geqq 1$ or $n=0$ and $X$ infinite.

Let $k \in N$. Then there exist $k$ disjoint closed subsets of $X$ with dimension $\geqq n$.

Proof. The proof is by techniques similar to those of C. H. Dowker [2] who proved related results.

If $n=0$ we use the fact that $X$ is infinite to prove our result. If $n>0$ the result will follow from: there exist two disjoint closed subsets of $X$ with dimension $\geqq n$. Let $\mathscr{U}=\left\{U_{1}, \cdots, U_{i}\right\}$ be an open cover which has no open shrinking of order $\leqq n$ and has no proper subcover. Since $n>0, i \geqq 2$. Let $\mathscr{F}=\left\{F_{1}, \cdots, F_{i}\right\}$ be a closed shrinking of $\mathscr{U}$. From [3, p. 276] it follows that some element of $\mathscr{F}$, say $F_{1}$, has dimension $\geqq n$. Let $V$ be an open set such that $F_{1} \subset V \subset \bar{V} \subset U_{1}$. We claim: $\operatorname{dim}(X \backslash V) \geqq n$. Indeed, the collection $\left\{U_{1}, U_{2} \backslash \bar{V}, \cdots, U_{i} \backslash \bar{V}\right\}$ is an open cover of $X \backslash V$. If $\operatorname{dim}(X \backslash V)<n$, by standard methods one can prove the existence of a collection $\mathscr{W}=$ $\left\{W_{1}, \cdots, W_{i}\right\}$ of open subsets of $X$ such that $W_{1} \subset U_{1}, W_{2} \subset U_{2} \backslash \bar{V}, \cdots$, $W_{i} \subset U_{i} \backslash \bar{V}$, ord $\mathscr{W} \leqq n$ and $X \backslash V \subset \cup \mathscr{W}$. Then define $O_{1}=V \cup W_{1}$, $O_{2}=W_{2}, \cdots, O_{i}=W_{i}$ to get an open cover $\left\{O_{1}, \cdots, O_{i}\right\}$ of $X$ with order $\leqq n$ and shrinking $\mathscr{C}$, contradicting our initial assumption. Thus $\operatorname{dim} F_{1} \geqq n, \operatorname{dim} X \backslash V \geqq n$, and $F_{1} \cap(X \backslash V)=\varnothing$. This proves Lemma 2 .

Proposition 2. Let $X$ be a normal space with $\operatorname{dim} X \geqq n$ and let either $n \geqq 1$ or $n=0$ and $X$ infinite. Let $k \in N$. Then

$$
\begin{array}{ll}
\Delta_{k}(X) \geqq 2^{k}-1 & \text { if } k \leqq n+1 \\
\Delta_{k}(X) \geqq\left(\begin{array}{l}
k \\
1
\end{array}\right)+\cdots+\left(\begin{array}{c}
k \\
n+1
\end{array}\right) & \text { if } k \geqq n+1 .
\end{array}
$$

Proof. We will only prove the proposition for the case $k \geqq n+1$, since the case $k \leqq n+1$ then follows by substituting $k-1$ for $n$. So, let $k \geqq n+1$. Let $\left\{C(\alpha) \mid \alpha \in C_{n+1}^{k}\right\}$ be a collection of disjoint 
closed subsets of $X$ of dimension $\geqq n$ (Lemma 2). For each $\alpha \in C_{n+1}^{k}$ we can find, by Lemma 1 , an open cover $\mathscr{C}(\alpha)=\left\{U_{i}^{\alpha} \mid i \in \alpha\right\}$ of $C(\alpha)$ which cannot be shrunk to a vanishing open cover of $C(\alpha)$. Note that $\left|\mathscr{U}_{(}(\alpha)\right|=n+1$. Note also that $\mathscr{C}(\alpha)$ cannot be shrunk to a vanishing closed cover of $C(\alpha)$ either, since such a cover could, by using normality of $C(\alpha)$, be expanded to a vanishing open cover of $C(\alpha)$ still refining $\mathscr{C}(\alpha)$. Now, define open subsets $U_{i}(1 \leqq i \leqq k)$ of $X$ as follows:

$$
U_{i}=\left[X \backslash \bigcup\left\{C(\alpha) \mid \alpha \in C_{n+1}^{k}\right] \cup\left[\bigcup\left\{U_{i}^{\alpha} \mid i \in \alpha\right\}\right]\right.
$$

(note that $\alpha$, not $i$, is the free variable in the right hand formula). Then $\mathscr{U}=\left\{U_{i} \mid 1 \leqq i \leqq k\right\}$ is an open cover of $X$. Suppose $\mathscr{V}^{4}<\mathscr{U}$ for a finite open cover $\mathscr{V}$. We claim: $|\mathscr{V}| \geqq\left(\begin{array}{c}k \\ 1\end{array}\right)+\cdots+\left(\begin{array}{c}k \\ n+1\end{array}\right)$, which implies $\Delta_{k}(X) \geqq\left(\begin{array}{l}k \\ 1\end{array}\right)+\cdots+\left(\begin{array}{c}k \\ n+1\end{array}\right)$. We will show this in the following way: let $\beta \subset\{1, \cdots, k\}$ be chosen so that $1 \leqq|\beta| \leqq$ $n+1$. We will prove the existence of an element $V(\beta) \in \mathscr{C}$ such that

$$
\beta=\left\{j \in\{1, \cdots, k\} \mid V(\beta) \subset U_{j}\right\} .
$$

In this way we can assign in a one-to-one manner an element $V(\beta) \in \mathscr{V}$ to every $\beta$. Since there are $\left(\begin{array}{l}k \\ 1\end{array}\right)+\cdots+\left(\begin{array}{c}k \\ n+1\end{array}\right) \beta$ 's, this gives us $|\mathscr{V}| \geqq\left(\begin{array}{l}k \\ 1\end{array}\right)+\cdots+\left(\begin{array}{c}k \\ n+1\end{array}\right)$. So, let $\beta \subset\{1, \cdots, k\}$ be subject to the condition $1 \leqq|\beta| \leqq n+1$, and fixed. Let $\gamma \subset\{1, \cdots, k\}$ be so that $\beta \cap \gamma=\varnothing$ and $\beta \cup \gamma \in C_{n+1}^{k}$. We will write $\alpha=\beta \cup \gamma$. Put $K=$ $C(\alpha) \backslash \bigcup\left\{U_{i}^{\alpha} \mid i \in \gamma\right\}$. Observe that $\left\{U_{j}^{\alpha} \mid j \in \beta\right\}$ is a collection of open subsets of $C(\alpha)$ which covers $K$ and cannot be shrunk to vanishing closed cover $\left\{K_{j} \mid j \in \beta\right\}$ on $K$ : namely, suppose it could. For each $j \in \beta$, the set $K_{j}$ could, by normality of $C(\alpha)$, be expanded to an open set $H_{j}$ of $C(\alpha)$ such that $K_{j} \subset H_{j} \subset U_{j}^{\alpha}$ in such a way that $\bigcap\left\{H_{j} \mid j \in \beta\right\}=\varnothing$. Thus $\mathscr{\mathscr { C }}(\alpha)=\left\{U_{i}^{\alpha} \mid i \in \alpha\right\}$ could be shrunk to the vanishing open cover $\left\{H_{j} \mid j \in \beta\right\} \cup\left\{U_{i}^{\alpha} \mid i \in \gamma\right\}$ of $C(\alpha)$, which is a contradiction. Now define closed sets $G_{j}, j \in \beta$, as follows.

For any $j \in \beta$, put $G_{j}=\left\{x \in K \mid \operatorname{St}(x, \mathscr{Y}) \subset U_{j}\right\}$. Then it is easy to see that $G_{j} \subset U_{j}^{\alpha}$ and that $\bigcup\left\{G_{j} \mid j \in \beta\right\}=K$ (recall that $\mathscr{V}^{\Delta}<\mathscr{U}$ ). Hence $\bigcap\left\{G_{j} \mid j \in \beta\right\} \neq \varnothing$. Let $x \in \bigcap\left\{G_{j} \mid j \in \beta\right\}$. Let $V(\beta)$ be an element of $\mathscr{C}$ containing $x$. Since $x \in G_{j}$ for $j \in \beta, V(\beta) \subset U_{j}$ for $j \in \beta$. Since $x \notin U_{i}$ for $\mathrm{i} \notin \beta, V(\beta) \not \subset U_{i}$ for $i \notin \beta$. Thus $\beta=$ $\left\{j \in\{1, \cdots, k\} \mid V(\beta) \subset U_{j}\right\}$. As noted above, this suffices to prove the proposition.

Combining Propositions 1 and 2, we have the following

CoRollary. Let $X$ be an infinite normal space, with $\operatorname{dim} X=$ 
$n, 0 \leqq n \leqq \infty$, and let $k$ be a natural number. Then

$$
\begin{array}{ll}
\Delta_{k}(X)=2^{k}-1 & \text { if } k \leqq n+1 \\
\Delta_{k}(X)=\left(\begin{array}{l}
k \\
1
\end{array}\right)+\cdots+\left(\begin{array}{c}
k \\
n+1
\end{array}\right) \text { if } k \geqq n+1 .
\end{array}
$$

REMARK. This corollary is nothing but a special case of our main theorem. The first equality holds for finite - as well for infinite dimensional $X$.

Proof. If $X$ is finite dimensional, this is a combination of Propositions 1 and 2. If $\operatorname{dim} X=\infty$, Proposition 2 gives us $A_{k}(X) \geqq$ $2^{k}-1$, thus we only have to prove $A_{k}(X) \leqq 2^{k}-1$. To this end, let $O=\left\{O_{1}, \cdots, O_{k}\right\}$ be an open cover. Obviously, ord $\circlearrowright \leqq k$. Now take this $O$ and substitute it for $\mathscr{C}$ in the proof of Proposition 1. Since in this proof the fact $\operatorname{dim} X \leqq n$ is used only to find this $\mathscr{Y}$ with ord $\mathscr{Y} \leqq n+1$, everything still works and we find a cover $\mathscr{H}^{\prime \prime}$ with $\left|\mathscr{V}^{-}\right| \leqq 2^{k}-1$ and $\mathscr{W}^{\lrcorner}<\mathscr{O}$. This proves our corollary.

4. The main theorem: the completely regular case. In this section we will extend the above result to the class of completely regular spaces. Let, for $X$ in this class, $\beta X$ be its Čech-Stone compactification.

Lemma 3. Let $X$ be a Tychonoff space. Then $\operatorname{dim} X=\operatorname{dim} \beta X$.

Proof. This is well-known and, in fact, may be chosen to be the definition of $\operatorname{dim} X$. See e.g., [3, p. 272].

Proposition 3. Let $X$ be a Tychonoff space, and $k \geqq 1$. Then $\Delta_{k}(X)=\Delta_{k}(\beta X)$.

Proof. Proposition 1, applied to $X$, and Proposition 2, applied to $\beta X$, together with Lemma 3 yield $\Delta_{k}(X) \leqq \Delta_{k}(\beta X)$. To prove the converse, let $k \in N$ and $\mathbb{Z}=\left\{U_{1}, \cdots, U_{k}\right\}$ be a functionally open cover of $\beta X$ such that for every (functionally) open cover $\mathscr{P}$ of $\beta X$ with $\mathscr{O}^{\Delta}<\mathscr{Z}$ the relation $|\mathscr{V}| \geqq \Delta_{k}(\beta X)$ holds true. Shrink $\mathscr{Z}_{6}$ to a functionally open cover $\mathscr{Z}^{\prime}=\left\{U_{1}^{\prime}, \cdots, U_{k}^{\prime}\right\}$ with $\bar{U}_{i}^{\prime} \subset U_{i}(1 \leqq i \leqq k)$. Define $\mathscr{W}=\left\{U_{1}^{\prime} \cap X, \cdots, U_{k}^{\prime} \cap X\right\}$. Then $\mathscr{V}$ is a functionally open cover of $X$. Suppose $\mathscr{W}^{\prime \prime}$ is a functionally open cover of $X$ with $\mathscr{W}^{\prime \prime}<\mathscr{W}$. We will prove: $\left|\mathscr{Y}^{\prime \prime}\right| \geqq \Delta_{k}(\beta X)$.

Let Ex be theop erator which assigns to every open subset $O$ of $X$ the largest open subset, $\operatorname{Ex} O$, of $\beta X$ with the property that $\operatorname{Ex} O \cap X=O$. In [3, p. 269-270] it is proved that $\operatorname{Ex}\left(O_{1} \cap O_{2}\right)=$ 
$\operatorname{Ex} O_{1} \cap \operatorname{Ex} O_{2}$ for open sets $O_{1}$ and $O_{2}$, and that $\operatorname{Ex}\left(O_{1} \cup O_{2}\right)=\operatorname{Ex} O_{1} \cup \operatorname{Ex} O_{2}$ whenever $O_{1}$ and $O_{2}$ are functionally open. Furthermore, it is easily seen that Ex $O \subset \bar{O}$ (closure taken in $\beta X$ ). Write $\mathscr{Y}^{-\prime}=\left\{W_{1}^{\prime}, \cdots, W_{l}^{\prime}\right\}$ and define $\mathscr{Y}=\left\{\operatorname{Ex} W_{1}^{\prime}, \cdots, \operatorname{Ex} W_{l}^{\prime}\right\}$. Then $\bigcup \mathscr{Y}=\operatorname{Ex} W_{1}^{\prime} \cup \cdots \cup \operatorname{Ex} W_{1}^{\prime}=$ $\operatorname{Ex}\left(W_{1}^{\prime} \cup \cdots \cup W_{l}^{\prime}\right)=\operatorname{Ex} X=\beta X$, thus $\mathscr{Y}$ is an open cover of $\beta X$. Let $p \in \beta X$ and consider all elements of $\mathscr{Y}$ that contain $p$.

Let us say that these are $\operatorname{Ex} W_{1}^{\prime}, \cdots, \operatorname{Ex} W_{m}^{\prime}(m \leqq l)$. Apparently, $\varnothing \neq \operatorname{Ex} W_{1}^{\prime} \cap \cdots \cap \operatorname{Ex} W_{m}^{\prime}=\operatorname{Ex}\left(W_{1}^{\prime} \cap \cdots \cap W_{m}^{\prime}\right)$, which implies $W_{1}^{\prime} \cap \cdots \cap W_{m}^{\prime} \neq \varnothing$. Let $q \in W_{1}^{\prime} \cap \cdots \cap W_{m}^{\prime}$. Since $\mathscr{W}^{\prime \Delta}<\mathscr{W}$, $W_{1}^{\prime} \cup \cdots \cup W_{m}^{\prime} \subset \operatorname{St}\left(q, \mathscr{V}^{\prime}\right) \subset U_{i}^{\prime} \cap X$ for some $i, 1 \leqq i \leqq k$. Thus $\operatorname{St}(p, \mathscr{V})=\operatorname{Ex} W_{1}^{\prime} \cup \cdots \cup \operatorname{Ex} W_{m}^{\prime}=\operatorname{Ex}\left(W_{1}^{\prime} \cup \cdots \cup W_{m}^{\prime}\right) \subset \operatorname{Ex}\left(U_{i}^{\prime} \cap X\right) \subset$ $\overline{U_{i}^{\prime} \cap X} \subset \bar{U}_{i}^{\prime} \subset U_{i} \in \mathscr{C}$. Therefore $\mathscr{V}^{\Delta}<\mathscr{H}$, and by the choice of $\mathscr{\mathscr { C }}$ and the fact that $|\mathscr{V}|=\left|\mathscr{V}^{\prime}\right|=l$ we conclude $l \geqq \Delta_{k}(\beta X)$. This proves $\Delta_{k}(X) \geqq \Delta_{k}(\beta X)$. Since we already had $\Delta_{k}(X) \leqq \Delta_{k}(\beta X)$, the proposition is proved.

Corollary. Let $X$ be an infinite Tychonoff space, and let $k$ be a natural number. Then, if $\operatorname{dim} X=n, 0 \leqq n \leqq \infty$,

$$
\begin{array}{ll}
\Delta_{k}(X)=2^{k}-1 & \text { if } k \leqq n+1 \\
\Delta_{k}(X)=\left(\begin{array}{l}
k \\
1
\end{array}\right)+\cdots+\left(\begin{array}{c}
k \\
n+1
\end{array}\right) \text { if } k \geqq n+1 .
\end{array}
$$

Proof. This follows immediately from Lemma 3, Proposition 3, and the corollary in the preceding section.

5. The main theorem: the general case. Let $X$ be a topological space. Define a completely regular space $\tilde{X}$ and a continuous mapping $\rho: X \rightarrow \widetilde{X}$ as follows: if $\mathscr{F}=\{f \mid f: X \rightarrow[0,1], f$ is continuous $\}$, let $\phi: X \rightarrow \Pi_{f \in \mathscr{T}} I_{f}$ be defined by $\phi(x)=(f(x))_{f \in \ldots}$. (Here $I_{f}=[0,1]$ for $f \in \mathscr{F}$.) Define $\widetilde{X}=\phi(X)$.

REMARK. The functor which associates $\tilde{X}$ with $X$ was used in dimension theory by $K$. Morita [7] under the name of Tychnoff functor.

Lemma 4. (i ) If $U \subset X$ is functionally open, so is $\phi(U) \subset \widetilde{X}$;

(ii) If $U \subset X$ is functionally open, then $U=\phi^{-1}(\dot{\phi}(U))$;

(iii) If $O_{1}$ and $O_{2} \subset X$ are functionally open, then $\dot{\phi}\left(O_{1} \cap O_{2}\right)=$ $\dot{\phi}\left(O_{1}\right) \cap \dot{\phi}\left(O_{2}\right)$.

Proof. (i) It suffices to observe, that if $U=f^{-1}((0,1]), \phi(U)=$ $\pi_{f}^{-1}((0,1])$, where $\pi_{f}: X \rightarrow I_{f}$ is projection; (ii) if $U=f^{-1}((0,1])$, and $x \notin U$, then $f(x)=0$, thus $x \notin \dot{\phi}^{-1}(\dot{\phi}(U))$, (iii) always $\dot{\phi}\left(O_{1} \cap O_{2}\right) \subset \dot{\phi}\left(O_{1}\right) \cap$ 
$\dot{\phi}\left(O_{2}\right)$. Let $p \in \phi\left(O_{1}\right) \cap \phi\left(O_{2}\right)$. Then there are $x \in O_{1}, y \in O_{2}$ with $\phi(x)=$ $\dot{\phi}(y)=p$. Thus $y \in \dot{\phi}^{-1}\left(\phi\left(O_{1}\right)\right)=O_{1}$.

Therefore $p=\dot{\phi}(y) \in \phi\left(O_{1} \cap O_{2}\right)$.

Proposition 4. (i) (K. Morita [7]) $\operatorname{dim} X=\operatorname{dim} \tilde{X}$;

(ii) $\Delta_{k}(X)=\Delta_{k}(\tilde{X})$ for all $k \in N$.

Proof. (i) Let $\operatorname{dim} X \leqq n$, and let $\mathscr{C}=\left\{U_{1}, \cdots, U_{m}\right\}$ be a functionally open cover of $\tilde{X}$. Obviously, $\mathscr{C}^{\prime}=\left\{\phi^{-1}\left(U_{1}\right), \cdots, \phi^{-1}\left(U_{m}\right)\right\}$ is a functionally open cover of $X$. Let $\mathscr{V}^{\prime}=\left\{V_{1}^{\prime}, \cdots, V_{l}^{\prime}\right\}$ be a functionally open refinement of $\mathscr{Q}^{\prime}$ such that ord $\mathscr{V}^{\prime} \leqq n+1$ and define $\mathscr{V}=\left\{\phi\left(V_{1}^{\prime}\right), \cdots, \phi\left(V_{l}^{\prime}\right)\right\} . \quad$ By (i) of the preceding lemma, $\mathscr{V}$ is a functionally open cover of $\widetilde{X}$ and $\mathscr{V}<\mathscr{C}$; from (iii) of the same lemma it follows that ord $\mathscr{V}=$ ord $\mathscr{V}^{\prime} \leqq n+1$. This proves $\operatorname{dim} X \leqq n$. Now let $\operatorname{dim} \tilde{X} \leqq n$, and let $\mathscr{U}=\left\{U_{1}, \cdots, U_{m}\right\}$ be a functionally open cover of $X$. Then $\mathscr{U}^{\prime}=\left\{\phi\left(U_{1}\right), \cdots, \phi\left(U_{m}\right)\right\}$ is a functionally open cover of $\tilde{X}$, and, consequently, has a functionally open refinement $\mathscr{V}^{\prime}=$ $\left\{V_{1}^{\prime}, \cdots, V_{l}^{\prime}\right\}$ with ord $\mathscr{V}^{\prime} \leqq n+1$. Now $\mathscr{V}=\left\{\dot{\phi}^{-1}\left(V_{1}^{\prime}\right), \cdots, \phi^{-1}\left(V_{l}^{\prime}\right)\right\}$ is a functionally open cover of $X$ with ord $\mathscr{V} \leqq n+1$. Take an element of $\mathscr{Y}$, e.g., $\phi^{-1}\left(V_{1}^{\prime}\right)$. There is some $i, 1 \leqq i \leqq m$, such that $V_{1}^{\prime} \subset \phi\left(U_{i}\right)$. But then $\phi^{-1}\left(V_{1}^{\prime}\right) \subset \phi^{-1}\left(\phi\left(U_{i}\right)\right)=U_{i}$, by (ii) of the proceding lemma. Thus $\mathscr{V}<\mathscr{C}$, which completes the proof of the first part of Proposition 4.

(ii) From (i), Proposition 1 and the corollary of $\S 4$ it follows that $\Delta_{k}(X) \leqq \Delta_{k}(\widetilde{X})$. To prove the converse, let $\mathscr{\mathscr { C }}=\left\{U_{1}, \cdots, U_{k}\right\}$ be a functionally open cover of $\tilde{X}$. Define $\mathscr{U}^{\prime}=\left\{\dot{\phi}^{-1}\left(U_{1}\right), \cdots, \phi^{-1}\left(U_{k}\right)\right\}$. Then there exists a functionally open cover $\mathscr{V}^{\prime}=\left\{V_{1}^{\prime}, \cdots, V_{m}^{\prime}\right\}$ of $X$ with $\mathscr{\mathscr { V }}^{\prime \Delta}<\mathscr{C}^{\prime}$ and $m=\left|\mathscr{V}^{\prime}\right| \leqq \Delta_{k}(X)$. Put $\mathscr{\mathscr { V }}=\left\{\dot{\phi}(V) \mid V \in \mathscr{V}^{\prime}\right\}$. Then $\mathscr{V}$ is a functionally open cover of $\tilde{X}$. Let $p \in \widetilde{X}$, and consider the elements of $\mathscr{V}$ which contain $p$. Let us say that these are $\phi\left(V_{1}^{\prime}\right)$, $\cdots, \phi\left(V_{l}^{\prime}\right)$. Since $\phi\left(V_{1}^{\prime}\right) \cap \cdots \cap \phi\left(V_{l}^{\prime}\right) \neq \varnothing$, we infer, by (iii) of Lemma $5, V_{1}^{\prime} \cap \cdots \cap V_{l}^{\prime} \neq \varnothing$. But $\mathscr{V}^{\prime\lrcorner}<\mathscr{U}^{\prime}$, so there exists $U_{i}^{\prime} \in \mathscr{U}_{i}^{\prime}$ with $V_{1}^{\prime} \cup \cdots \cup V_{l}^{\prime} \subset U_{i}^{\prime}$. Therefore $\phi\left(V_{1}^{\prime}\right) \cup \cdots \cup \phi\left(V_{l}^{\prime}\right)=\phi\left(V_{1}^{\prime} \cup \cdots \cup V_{l}^{\prime}\right) \subset$ $\dot{\phi}\left(U_{i}^{\prime}\right)=U_{i}$. Thus $\mathscr{V}^{4}<\mathscr{U}$, and $|\mathscr{V}| \leqq\left|\mathscr{V}^{\prime}\right|=m \leqq \Delta_{k}(X)$. This proves $\Delta_{k}(\widetilde{X}) \leqq \Delta_{k}(X)$, and completes the proof of Proposition 4 .

Now we will state and prove our

MAIN THEOREM. Let $X$ be a topological space, such that either $1 \leqq \operatorname{dim} X \leqq \infty$ or $\operatorname{dim} X=0$ and $\widetilde{X}$ is infinite. Let $\operatorname{dim} X=n$, and let $k$ be a natural number. Then

$$
\begin{array}{ll}
\Delta_{k}(X)=2^{k}-1 & \text { if } k \leqq n+1 \\
\Delta_{k}(X)=\left(\begin{array}{l}
k \\
1
\end{array}\right)+\cdots+\left(\begin{array}{c}
k \\
n+1
\end{array}\right) \text { if } k \geqq n+1 .
\end{array}
$$


Proof. Note that the conditions of the theorem imply that $\tilde{X}$ is always infinite: for if $\operatorname{dim} X>0$, so is $\operatorname{dim} \tilde{X}$ by Proposition 4, and a Tychonoff space with positive dimension is always infinite. So we may apply the corollary of $\S 4$ to $\tilde{X}$, and this, together with Proposition 4, proves our result.

CoRollary. If $X$ satisfies the conditions of the main theorem,

$$
\operatorname{dim} X=\lim _{k \rightarrow \infty} \frac{\log \Delta_{k}(X)}{\log k}-1 \text {. }
$$

\section{REFERENCES}

1. J. Bruijning, A characterization of dimension of topological spaces by totally bounded pseudometrics, to appear.

2. C. H. Dowker, Local dimension of normal spaces, Quart. J. Math. Oxford, 6 (1955), 101-120.

3. R. Engelking, Outline of general topology, (North-Holland, Amsterdam, 1968).

4. J. Nagata, Modern dimension theory, (North-Holland, Amsterdam, 1964).

5. - Modern general topology, (North-Holland, Amsterdam, 1974).

6. L. Pontrjagin and L. Schnirelmann, Sur une propriété métrique de la dimension, Ann. of Math., (2) 33 (1932), 152-162.

7. K. Morita, Čech cohomology and covering dimension for topological spaces, Fund. Math., 87 (1975), 31-52.

Received March 3, 1978.

UNIVERSITY OF AMSTERDAM

ROETERSSTRAAT 15

AMSTERDAM 1004, HolLAND 


\section{PACIFIC JOURNAL OF MATHEMATICS}

\section{EDITORS}

RICHARD ARENS (Managing Editor)

University of California

Los Angeles, California 90024

C. W. CURTIS

University of Oregon

Eugene, OR 97403

C. C. MOORE

University of California

Berkeley, CA 94720
J. DugundjI

Department of Mathematics University of Southern Californı Los Angeles, California 90007

R. Finn and J. Milgram Stanford University Stanford, California 94305

ASSOCIATE EDITORS

E. F. BECKENBACH

B. H. NeUmanN

F. WOLF

K. YoSHIDA

\section{SUPPORTING INSTITUTIONS}

UNIVERSITY OF BRITISH COLUMBIA CALIFORNIA INSTITUTE OF TECHNOLOGY

UNIVERSITY OF CALIFORNIA

MONTANA STATE UNIVERSITY

UNIVERSITY OF NEVADA, RENO

NEW MEXICO STATE UNIVERSITY

OREGON STATE UNIVERSITY

UNIVERSITY OF OREGON
UNIVERSITY OF SOUTHERN CALIFORNIA

STANFORD UNIVERSITY

UNIVERSITY OF HAWAII

UNIVERSITY OF TOKYO

UNIVERSITY OF UTAH

WASHINGTON STATE UNIVERSITY

UNIVERSITY OF WASHINGTON 


\section{Pacific Journal of Mathematics}

\section{Vol. 80, No. $1 \quad$ September, 1979}

Jeroen Bruijning and Jun-iti Nagata, A characterization of covering dimension by

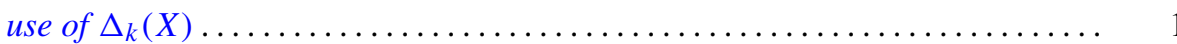

John J. Buoni and Albert Jonathan Klein, On the generalized Calkin algebra ...... 9

Thomas Ashland Chapman, Homotopy conditions which detect simple homotopy

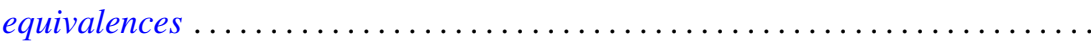

John Albert Chatfield, Solution for an integral equation with continuous interval

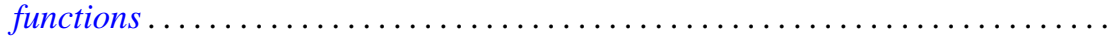

Ajit Kaur Chilana and Ajay Kumar, Spectral synthesis in Segal algebras on

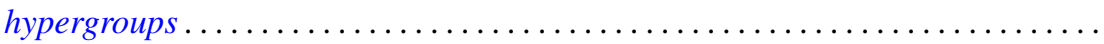

Lung O. Chung, Jiang Luh and Anthony N. Richoux, Derivations and

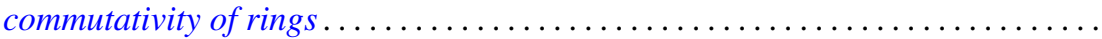

Michael George Cowling and Paul Rodway, Restrictions of certain function spaces to closed subgroups of locally compact groups .....................

David Dixon, The fundamental divisor of normal double points of surfaces........

Hans Georg Feichtinger, Colin C. Graham and Eric Howard Lakien,

Nonfactorization in commutative, weakly selfadjoint Banach algebras . . . . . . .

Michael Freedman, Cancelling 1-handles and some topological imbeddings ....... .

Frank E., III Gerth, The Iwasawa invariant $\mu$ for quadratic fields . . . . . . . . . . . . . .

Maurice Gilmore, Three-dimensional open books constructed from the identity

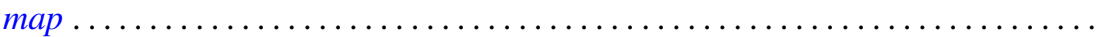

Stanley P. Gudder, A Radon-Nikodým theorem for $*$-algebras .

Peter Wamer Harley, III and George Frank McNulty, When is a point Borel? .

Charles Henry Heiberg, Fourier series with bounded convolution powers . .

Rebecca A. Herb, Characters of averaged discrete series on semisimple real Lie

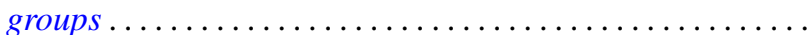

Hideo Imai, On singular indices of rotation free densities . .

Sushil Jajodia, On 2-dimensional CW-complexes with a single 2-cell . . .

Herbert Meyer Kamowitz, Compact operators of the form $u C_{\varphi}$

Matthew Liu and Billy E. Rhoades, Some properties of the Chebyshev method...

213

George Edgar Parker, Semigroups of continuous transformations and generating

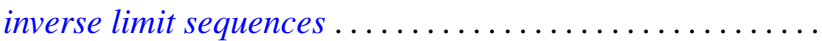

Samuel Murray Rankin, III, Oscillation results for a nonhomogeneous

equation ...

Martin Scharlemann, Transverse Whitehead triangulations ...

Gary Joseph Sherman, A lower bound for the number of conjugacy classes in a

finite nilpotent group

Richard Arthur Shoop, The Lebesgue constants for $\left(f, d_{n}\right)$-summability .

Stuart Jay Sidney, Functions which operate on the real part of a uniform

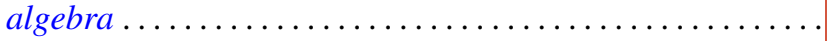

Tim Eden Traynor, The group-valued Lebesgue decomposition 